\title{
Powering for Long Term Monitoring in the Enclosed Areas
}

\author{
A. Boura, M. Husak \\ Department of Microelectronics, Faculty of Electrical Engineering \\ Czech Technical University in Prague \\ Technicka 2, CZ - 16627 Prague 6, Czech Republic \\ Phone/Fax number: +420224352 237 / +420 224310 792, e-mail: bouraa@fel.cvut.cz, husak@fel.cvut.cz
}

\begin{abstract}
Paper presents possible solution and practical examples for wireless powering and signal transfer using the inductive coupling. It can be suitable for long term monitoring in enclosed areas and systems that are isolated form the surroundings. This solution allows avoiding the batteries. It can be used for probes bricked in to the wall, extreme temperature environment measurement, the barrels internal shells health monitoring etc. Main advantage of this kind of powering is nearly unlimited lifetime, no toxic pollutions (such as $\mathrm{Cd}, \mathrm{Li}$, $\mathrm{H}_{2} \mathrm{SO}_{4}, \ldots$ contained in the batteries) and universal applicability. The powering is provided by the near magnetic field. The resonance is considered in the design for increasing the voltage transfer. Also the passive signal transfer can be performed using this principle while it is similar to the RFID systems (Radio Frequency Identification). The main contribution of this paper is prediction of the powering potentialities and distance limits of this strategy. Voltage levels, power transfer efficiency and signal modulation effects are studied up to distance of $30 \mathrm{~cm}$. The simple coils are considered. Also special converter principle is presented for this purpose.
\end{abstract}

\section{Key words}

Wireless powering, long-term monitoring, enclosed systems measurement, passive signal transfer

\section{Introduction}

Wireless measurements are important in situations where the standard techniques can not be used. Wireless solution requires the special way of powering. Most used technique is to power the circuits using some kind of battery. The battery operation of the system has its limits caused namely by the limited lifetime, toxicity of the battery or an extreme temperatures environment that is improper for the batteries. There are several alternative possibilities how to power the isolated system but sometimes the energy must be wirelessly injected to the system. Method, which is described in this paper, injects the energy to the system and it is also proper for the data transfer. Most known application of this method are the RFID systems, where the chip containing the identification tag it is powered. This paper presents possibilities and limits of the powering via the inductive coupling where the serial and parallel resonance is used for maximization the transfer efficiency. Special kind of converter is presented which allows measurement and direct modulation of the transfer signal. The converter functionality is presented on example where the barrel internal shell integrity is monitored.

The converter is designed for fabrication using the standard CMOS technology while it is not realized yet. The measurements are performed on the circuit consisting of discrete devices.

\section{Model of the Inductive Coupling}

When two or more inductances partly share the magnetic field, the coupling is presented. Figure 1 presents an example of such coupling for arbitrary inductances. Voltage transferred to the second inductance can be calculated using the Faraday's law.

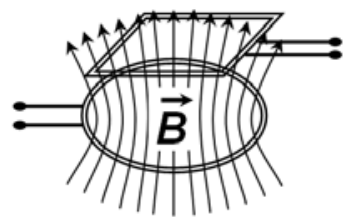

Fig. 1. Example of coupled inductances

The secondary voltage thus depends on the secondary coils geometry and on the magnetic field distribution of the primary inductance.

\section{A Coupling Coefficient}

In terms of the circuit theory the inductance coupling can be described using the mutual inductance or using the coupling coefficient. This approach is suitable because the standard simulation tools can be used for a description of the powering. As well as the voltage transfer also the coupling coefficient is given by the coils geometry and the magnetic field distribution. Most important topology of the inductances is the axial orientation from the Fig. 1. Coefficients dependency versus the distance is given by the magnetic field intensity distribution.

Circular coil of radius $r$, winding number $N$ and current carrying $I$, induces the magnetic field inductance $B$ (in 
environment of permeability $\mu_{0}$ ). The inductance versus the axial distance $x$ can be expressed using the equation (1) [1].

$$
B=\left.\frac{\mu_{0} \cdot N \cdot I \cdot r^{2}}{2 \cdot\left(r^{2}+x^{2}\right)^{\frac{2}{3}}} \approx \frac{\mu_{0} \cdot N \cdot I \cdot r^{2}}{2} \frac{1}{x^{3}}\right|_{x>>r}
$$

The field drop is proportional to $1 / x^{3}$ for distances much bigger than the coils diameter and thus also $k$ is dependent this way. For smaller distances the secondary coil is embracing also the non-axial magnetic field. This is why the coupling coefficient is changing faster for the smaller distances. This change is approximately exponential. Fig. 2 shows the measurement result of the coupling coefficient between the circular coil and the rectangular surface coil. This dependency is approximated using the exponential and the $1 / x^{3}$ functions.

Character of this dependency is changing approximately at the distance equal to the diameter of the bigger coil (vertical dash line in the picture). This knowledge of the coefficients character can be used for a prediction of the coefficients value for the other coils.

\section{B Circuit Behavior}

The mutual inductances from the Fig. 1 can be described using the equations that relate the voltage phasors $V_{1}, V_{2}$, current phasors $I_{1}, I_{2}$, and loading impedance $Z_{L}$ for the angular frequency $j \omega$. These equations can be used for derivation of the basic relationships in the circuit [2].

The most important parameter of the coupled inductances is the voltage transfer (2) and the input impedance which can be seen on the inductor $L_{l}(3)$ [2].

$$
\begin{gathered}
\hat{V}_{2}=\hat{V}_{1} \cdot \frac{k \cdot \hat{Z}_{L} \cdot \sqrt{\frac{L_{2}}{L_{1}}}}{\hat{Z}_{L}+j \omega L_{2} \cdot\left(1-k^{2}\right)} \\
\hat{Z}_{\text {in }}=\frac{\hat{V}_{1}}{\hat{I}_{1}}=\frac{j \omega L_{1} \cdot \hat{Z}_{L}+j \omega L_{1} \cdot j \omega L_{2} \cdot\left(1-k^{2}\right)}{\hat{Z}_{L}+j \omega L_{2}}
\end{gathered}
$$

The impedance depends on the loading impedance $Z_{L}$ and on the coupling coefficient $k$.

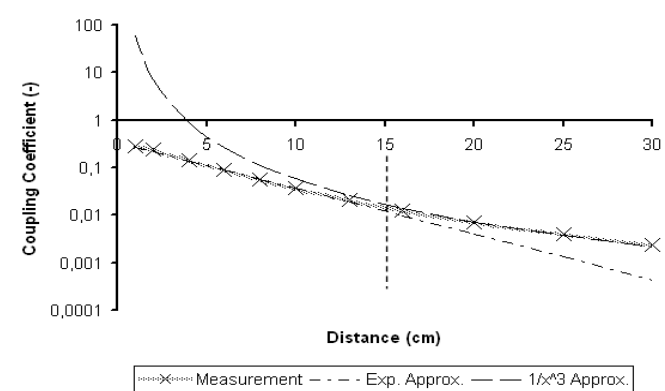

Fig. 2. Approximation by the exponential and $1 / x^{3}$ functions for the coupled coils
The transformation of the $Z_{L}$ to the input side is significant especially in resonance and it can be used for signal transfer (chapter 4).

\section{Equivalent Circuit}

For an understanding the effects in the circuit it is helpful to derive an equivalent circuit (Fig. 3) of the coupled inductances. Derivation of this circuit can be realized by observing the circuit behavior under the loading impedance change [3].

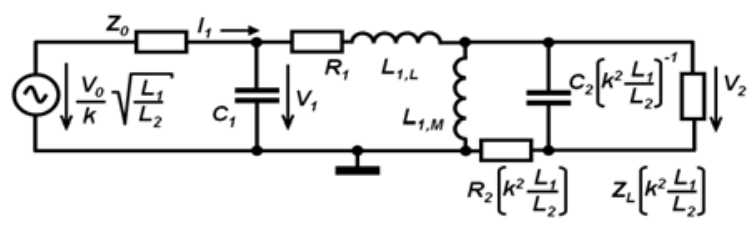

Fig. 3. Equivalent circuit respecting the parasitic properties of the inductances

First, the secondary side can be presumed to be shortcircuit $\left(Z_{L} \rightarrow 0\right)$. According the (3) the input impedance is equal to (4) which represents an inductance $L_{l, L}$.

$$
\lim _{Z_{L} \rightarrow 0} \hat{Z}_{i n}=j \omega L_{1} \cdot\left(1-k^{2}\right)=j \omega L_{1, L}
$$

This inductance is called the leakage inductance and it is characterized by the magnetic field excluding the secondary inductance. The magnetic field which contributes to the secondary side is represented by the magnetizing inductance $L_{l, M}$ - equation (5) [2].

$$
\begin{gathered}
L_{1, M}=L_{1} \cdot k^{2} \\
\hat{Z}_{L, e}=\hat{Z}_{L} \cdot \frac{L_{1}}{L_{2}} \cdot k^{2}
\end{gathered}
$$

Equation (6) represents the transformed loading impedance. Equivalent circuit thus consists of serial combination of leakage inductance and magnetizing inductances in parallel with the transformed loading impedance. Figure 3 shows the equivalent circuit including the parasitic properties of the inductances such as the serial resistances $R_{l}, R_{2}$ and parasitic capacitances $C_{1}, C_{2}$. Also the input voltage must be transformed in order to keep the circuit equivalent.

\section{Resonance Behavior}

It is evident from the Fig. 3, that the circuit exhibits some resonant behavior. Omitting the parasitic effects, the parallel resonance frequency $f_{r p 0}$ can be expressed by the (7). The inductance $L_{e}$ is a parallel combination of the $L_{l, L}$ and $L_{l, M}$. Parallel capacitance $C_{p}$ is the secondary side capacitance transformed using the (6).

$$
f_{r p 0}=\frac{1}{2 \cdot \pi \sqrt{L_{e} C_{p}}}=\frac{1}{2 \cdot \pi \sqrt{L_{2} \cdot C_{2} \cdot\left(1-k^{2}\right)}}
$$




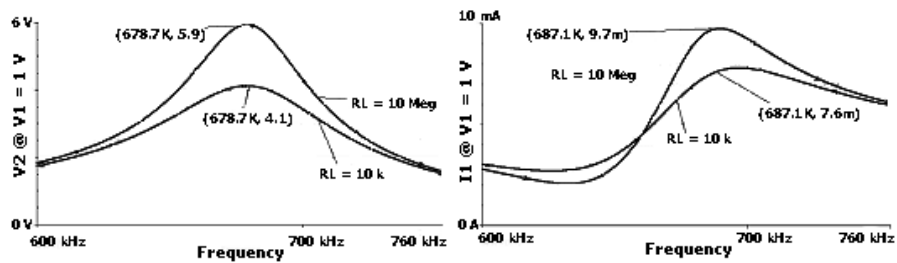

Fig. 4. Simulation according the Fig. 3, parallel resonance; $\mathrm{L} 1=55 \mu \mathrm{H}, \mathrm{L}_{2}=82 \mu \mathrm{H}, \mathrm{C}_{1}=150 \mathrm{pF}, \mathrm{R}_{1}=1.2 \Omega, \mathrm{R}_{2}=21.4 \Omega$, $\mathrm{k}=0.34 ; \mathrm{C} 2=758.4 \mathrm{pF}$

The voltage transfer in resonance can exceed the nominal transfer (2) (up to over thousands of percents) - see graph on the Fig. 4. This effect is crucial part of the powering strategy because the voltage level on the load must be high enough for rectification.

Also the serial resonance on the input can be presented (impedance $Z_{0}$ from the figure 3 must be the capacitance). This resonance increases the voltage transfer and it is more powerful for longer distances. Combination of both resonances is most suitable for long distance powering [5].

\section{Principle of Communication}

The voltage transfer in resonance is very sensitive on the loading impedance because it is changing the quality factor of the resonance tank circuit. Also the input current of the inductance $L_{l}$ is changing according the load (fig. 4). This effect can be used for communication between the powered probe and the transmitting device. Simple powering and communication scheme is presented on the fig. 5 .

The signal transfer from the sensing probe can be realized using the current measurement in the primary inductance. For each position of the switch it is different also the input current because the modulating resistor $R_{m}$ changes the quality factor. Loading impedance represented by the sensing probe is stable (or slowly changing) because of the filtering capacitor $C_{f}$. Current change on the input is thus caused mainly by the output signal from the probe.

Figure 6 presents example of signal transfer according the presented scheme. Upper side of the figure presents the input current $I_{\text {in }}$ and the bottom curve is the filtered voltage which can be used for the powering. Presented amplitude changes of the signals are caused by the switched $R_{m}$ of value $10 \mathrm{k} \Omega$.

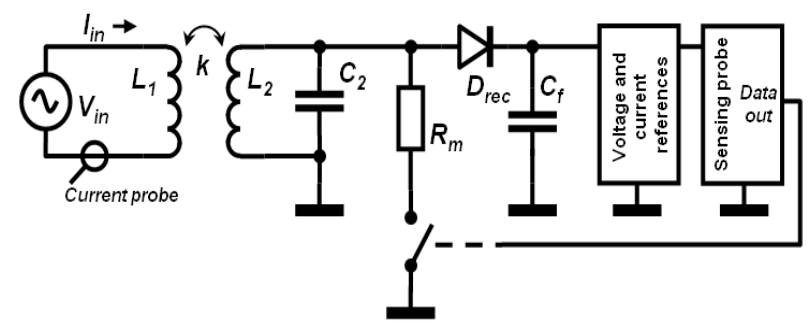

Fig. 5. Simplified powering and communication scheme

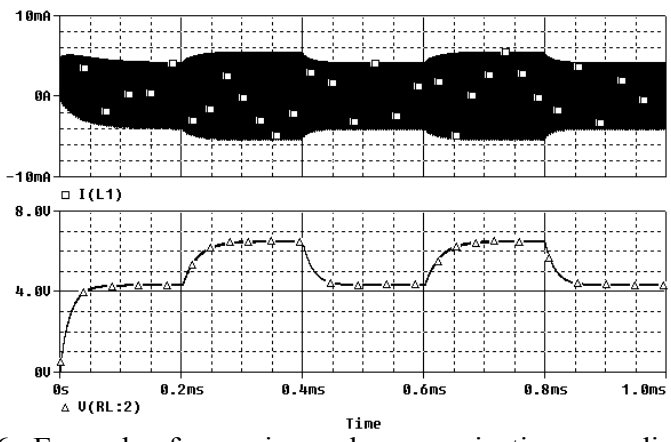

Fig. 6. Example of powering and communication according the figure 4

Frequency of the input source must be tuned according the (7). For given inductances and given frequency it can be tuned using the capacitance $C_{2}$. This capacitance is affected by the parasitic properties of the rectifying diode $D_{\text {rec }}$ and capacity of the switch. It is advisable to use high frequency and low voltage-drop devices for this purpose such a Schottky diode for rectifying and bipolar junction transistor as the switch. Filtering capacitor $C_{f}$ serves as the energy storage and its value does not affect the resonance frequency.

\section{Signal Converter}

According the presented communication scheme it is necessary to have a rectangular output from the sensing probe in order to switch the modulating resistor on the secondary inductance. Standard analog to digital converters (ADC) cannot be used for this purpose because of the power consumption and complexity of data output. It is necessary to design a simple converter with specific signal output. Possible solution is presented on the fig. 7.

Principle of this converter is based on a dual-slope ADC. Input current $I_{\text {in }}$ is integrated by a capacitor and when its voltage exceeds the specific level it changes the current flow and the capacitor is discharged by the reference current $I_{o}$. Output signal is represented by the signal which is changing the direction of the currents. Charging and discharging time ratio is proportional to the ratio of the input and the reference currents. Figure 8 presents possible output rectangular signal and signal presented on the charging capacitor.

This solution does not need any supporting circuit such a timing clock signal or combinatorial logic for data transfer management. Dynamic range of the converter is suitable for small input signals.

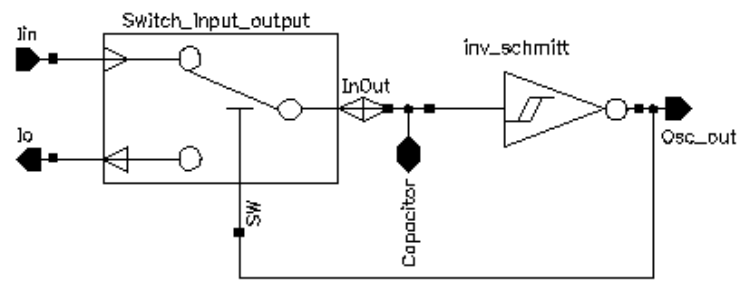

Fig. 7. Block scheme of the converter 


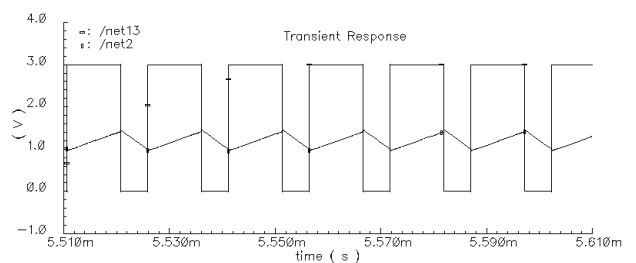

Fig. 8. Simulation result of the converter according the figure 7

Output pulse ratio can vary from 100 to 0.01 without any significant error. For bigger input signals the pulse width can be shorter than the transient performance of the signal modulation and thus it can be difficult for detection (see fig. 6). It is necessary to match the integrating capacitance to expected input and reference currents with respect to the dynamic behavior of the modulation.

\section{Measurement Results}

\section{A Powering Potential}

Figure 9 presents the measurement results for coupled inductances. As the primary inductance it is considered a simply winded coil of diameter $14 \mathrm{~cm}$ and winding number 10. Secondary inductance is the surface rectangular coil performed on the cuprextit board with the maximal side length of $9 \mathrm{~cm}$, winding number 40 and loop spacing $1 \mathrm{~mm}$ (see also figure 10). Properties of the inductances are same as the inductances used for the measurements and simulations from the figures 2 and 4 .

Voltage transfer and maximal theoretical power transfer efficiency is presented. The efficiency is expressed for ideally adapted load and it is given namely by the parasitic properties of the inductances and by the losses in the surroundings.

The frequency for measurement was chosen in order to keep the circuit in resonance. For parallel resonance it was slightly changed with the $k$ change according (7).

\section{B Application of the Converter for Monitoring}

Figure 10 shows the practical realization of the circuit from the figure 7 . It consists of the transistors from the integrated circuit HEF 4007. Similar circuit can be realized in the single chip.

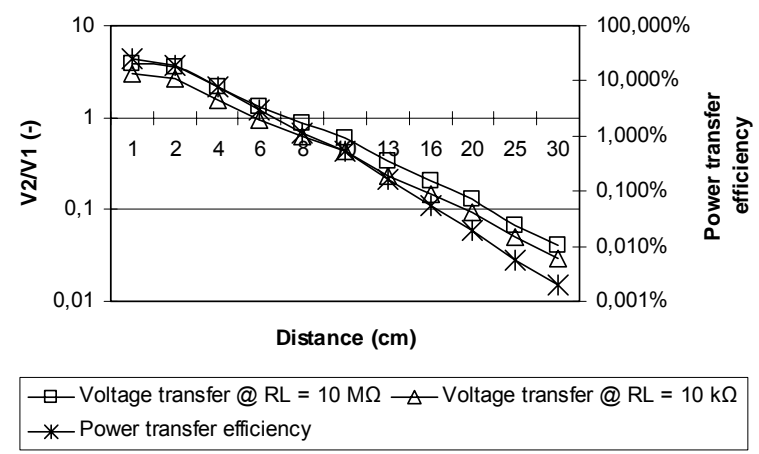

Fig. 9. Transfer for given inductances and parallel resonance
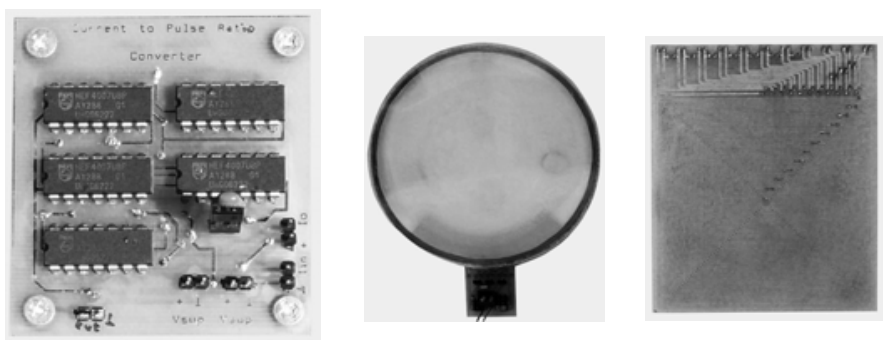

Fig. 10. Realized converter using the five integrated circuits HEF 4007 and coils used for powering and communication

This circuit was used in experiment which simulated the integrity monitoring of some kind of the surface (e.g. internal shell of the barrel). The circuit was connected according the scheme from the figure 11 .

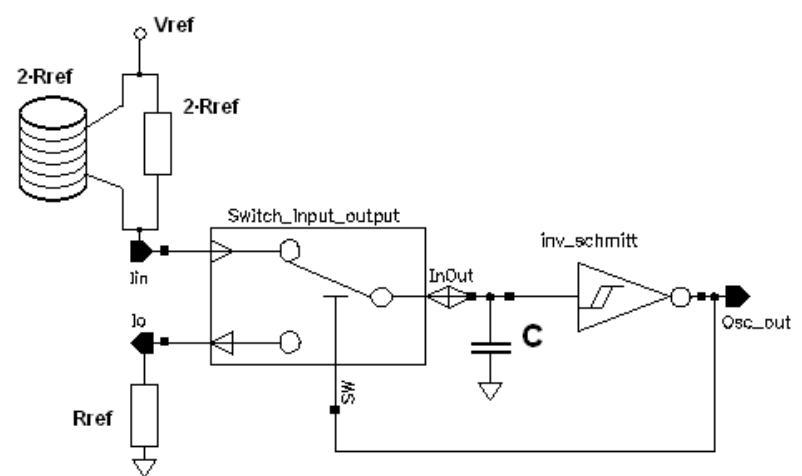

Fig. 11. Circuit connection for long-term monitoring of the barrel surface integrity

The input and the reference currents for charging the capacitance are dependent on the reference resistances Rref and the reference voltage Vref. In this situation the charging and discharging currents are not constant, (they are exponential) while for narrow decision voltage levels in the Schmitt trigger inverter the current can be considered nearly constant. The output signal pulse ratio is thus also constant.

One part of the charging reference resistance (Iin) can be represented by some sensing structure such as long and thin wire winded around the barrel. Interrupting the wire (mechanically or by the corrosion) the output signal pulse ratio changes and it can be easily detected by the input current change (chapter 4 ).

It is necessary to have two resistors in the charging branch in order to keep the circuit oscillating also when the wire is broken. The other resistors can be integrated directly on the chip.

If the wire is under corrosion, the resistance is changing continuously and also the output signal pulse ratio changes slowly. It can be performed statistical evaluation of the pulse ratio change and thus alert the problems in advance.

According the experiment the wire health monitoring can be performed up to distance of $20 \mathrm{~cm}$. 


\section{Conclusion}

Presented paper summarizes basic theory of the noideally coupled inductances, which can be used for powering the micro-systems. Measurements in real application were performed for surface rectangular coil on the secondary side and for the simple winded coil on the primary side. Parallel resonance was used for maximization the voltage transfer with respect to the possible power reliability and thus the frequency must be tuned properly. Different resonances can be also used. The parallel resonance is effective only for short distance powering. This configuration exhibits excellent sensitivity of the input current on the loading impedance so it is suitable also for communication with the powered device. Serial resonance is most powerful for the longer distances but it is not suitable for communication with the powered device (small dependency of the input current). Short distance potential can be an advantage in situations where the several measurements must be performed near together.

Special kind of converter was presented. It can be used for measurements in the enclosed systems. Its functionality was performed on example of surface health monitoring. This can be used as a safeguard for barrels containing dangerous pollutions. The output is represented by the rectangular signal with variable pulse ratio so it can be used also for other measurements.

\section{Acknowledgement}

Research described in the paper has been supported by the Czech Science Foundation project No. 102/09/1601 "Micro- and nano-sensor structures and systems with embedded intelligence" and partially by the research program No. MSM 6840770012 "Transdisciplinary
Research in Biomedical Engineering II" of the CTU in Prague.

\section{References}

[1] T. Prochazka, "Antennas for RFID systems", Elektrorevue, 2002, vol. 22, electronic file available at http://www.elektrorevue.cz/clanky/02022/index.html, 2009.

[2] A. Boura, P. Kulha, M. Husak, "Simple Wireless A/D Converter for Isolated Systems", IEEE ISIE 2009 Proceedings [CD-ROM], Seoul, Korea, p. 323 - 328, ISBN: 978-1-4244-4349-9

[3] Wu, J., Quinn, V., Bernstein, G. "A simple, wireless powering scheme for MEMS devices", MEMS Components and Applications for Industry, Automobiles Aerospace, and Communications, Proceedings of SPIE, 2001, Vol. 4559, URL:

http://www.ece.utk.edu/ jaynewu/Papers/SPIE\%202001\% 204559_7.pdf

[4] Kubar, M., Jakovenko, J.: "Tutorial of the Control Logic Design for Analogue Integrated Circuit Designers", Proc. of SMACD'08, Erfurt, Germany, pp. 237-240, 2008, ISBN: 978-3-00-025761-2

[5] Bouřa, A., Kulha, P., Husak, M.: "Applicability of the Inductive Powering for Enclosed Systems and Data Transfer", Proc. of $45^{\text {th }}$ international conference on microelectronics, devices and materials MIDEM 2009, Postojna, Slovenia, 2009, p.141-146, ISBN 978-96191023-9-8

[6] Popovic, Z.: "Wireless Powering for Low-Power Distributed Sensors", SERBIAN JOURNAL OF ELECTRICAL ENGINEERING, Vol. 3, No. 2, November 2006, p. 149-162

[7] Suster, M., Chaimanonart, N., Guo, J., Ko, W., Young, D. "Remote-Powered High-Performance Strain Sensing Microsystem", Micro Electro Mechanical Systems, MEMS 2005. 18th IEEE International Conference on, 30 Jan.-3 Feb. 2005 , p. $255-258$ 